\title{
Integración del sistema de gestión de la seguridad y salud en el trabajo en el sistema de gestión de calidad en las entidades públicas colombianas de orden nacional"
}

Integration of occupational health and safety management system in the quality management system in Colombian public entities of national level

Inteǵração do Sistemas de gestão da segurança e saúde no trabalho no sistema de gestão da qualidade em entidades públicas colombianas de ordem nacional

Recibido: 18 de diciembre de 2017

Revisado: 15 de enero de 2018

Aceptado: 21 de marzo de 2018

Daissy Carolina Peralta Crus**

Ministerio de Ambiente y Desarrollo Sostenible

Schneider Guataqui Cervera ${ }^{* *}$

Ministerio de Trabajo

Artículo de resultado de investigación. DOI: http://dx.doi.org/10.15332/s2145-1389.2018.0001.02

** Magíster en Calidad y Gestión Integral. Ingeniera ambiental. Coordinadora del grupo del sistema integrado de gestión. Ministerio de Ambiente y Desarrollo Sostenible. Correo electrónico: caroperaltacr@gmail.com

*** Magíster en Calidad y Gestión Integral. Especialista en Gerencia de la Seguridad y Salud en el Trabajo. Ingeniero industrial. Profesional especializado. Ministerio de Trabajo. Correo electrónico: ingschneiderg@gmail.com 


\section{RESUMEN}

En este artículo se presentan los resultados de una investigación en la cual se propone una metodología de integración del sistema de gestión de la seguridad y salud en el trabajo (SG-SST) en el sistema de gestión de calidad (SGC) en las entidades públicas colombianas de orden nacional. Lo anterior, dado la obligatoriedad de su implementación establecida en el Decreto 1072 de 2015 para todos los empleadores públicos y privados, y la Ley 872 de 2003 que establece el SGC en los organismos y entidades por servicios de la rama ejecutiva del poder público del orden nacional, entre otras. La investigación se desarrolló en cuatro etapas: revisión de la literatura identificando herramientas, estrategias y referentes teóricos de integración y validación; análisis de correspondencia de los requisitos integrables; elaboración de la propuesta metodológica estructurada con base en las directrices de la ILO-OSH-2001 y la validación de contenido de la propuesta con expertos. De acuerdo con lo anterior, esta metodología se convierte en una herramienta orientadora que brinda beneficios en la gestión institucional como controles integrados, mejora en la prestación del servicio, reducción de las interrupciones en el trabajo, prevención de sobrecostos y sanciones administrativas.

Palabras clave: seguridad y salud en el trabajo, calidad, sistema integrado de gestión, ISO 9001:2015, Decreto 1072 de 2015.

\section{ABSTRACT}

This article presents the results of a research project that proposes a methodology for integrating the occupational health and safety management system (OHSMS) into the quality management system (QMS) in Colombian public entities of national level. The foregoing, given the compulsory nature of its implementation established in Decree 1072 of 2015 for all public and private employers and Law 872 of 2003 establishes the QMS in the agencies and entities for services of the Executive Branch of the Public Power of the National level, among other. The research was developed in four stages: review of the literature identifying tools, strategies and theoretical references of integration and validation; correspondence analysis of the integrable requirements; preparation of the structured methodological proposal based on the ILOOSH-2001 guidelines and the validation of the content of the proposal with experts. In accordance with the above, this methodology becomes a guiding tool that provides benefits in institutional management such as integrated controls, improvement in service provision, reduction of work interruptions, prevention of over costs and administrative penalties.

Keywords: Occupational Health and Safety, Quality, Integrated Management System, ISO 9001:2015, Decree 1072 of 2015.

\section{RESUMO}

Neste artigo se apresentam os resultados de uma investigação na qual se propõe uma metodologia de integração do sistema de gestão da segurança e saúde no trabalho (SGSST) no sistema de gestão da qualidade (SGQ) nas entidades públicas colombianas de ordem nacional. $\mathrm{O}$ anterior, dada a obrigatoriedade de sua implementação estabelecida no Decreto 1072 de 2015 para todos os empregadores públicos e privados e a Lei 872 de 2003 estabelece o SGQ nos organismos e entidades por serviços do Poder Executivo da ordem nacional, entre outras. A investigação se desenvolveu em quatro etapas: revisão da literatura identificando ferramentas, estratégias e referentes teóricos de integração e validação; análise de correspondência dos requisitos integrais; elaboração da proposta metodológica 
estruturada com base nas diretrizes do ILO-OSH-2001 e validação do conteúdo da proposta com especialistas. De acordo com o anterior, Esta metodologia se converte em uma ferramenta orientadora que proporciona benefícios na gestão institucional como controles integrados, melhoria na prestação de serviços, redução das interrupções no trabalho, prevenção de sobre custos e sanções administrativas.

Palavras-chave: segurança e saúde no trabalho, qualidade, sistema integrado de gestão, ISO 9001:2015, Decreto 1072 de 2015.

\section{INTRODUCCIÓN}

Las consideraciones de seguridad, humanitarias, políticas y económicas, impulsan la creación de la Organización Internacional del Trabajo (OIT) en 1919, quien emite las directrices relativas a los sistemas de gestión de la seguridad y salud de los trabajadores ILOOSH 2001 (Organizacion Internacional del Trabajo, 2011). Por otro lado, en 1999 organismos certificadores crean la norma OHSAS 18001 actualizada en el 2007.

Los costos relacionados con la salud y la seguridad en el trabajo, según Gómez (2006), son muy representativos y de acuerdo a precisiones emitidas por la OIT, el $4 \%$ por ciento del PIB mundial están relacionadas con pérdidas por interrupciones en el trabajo, capacitación y formación; también cabe resaltar que alrededor de 2 millones de trabajadores fallecen por la ocurrencia de accidentes y enfermedades laborales, sin incluir los 270 millones de accidentes y 160 millones de enfermedades por ocasión al trabajo.

Por lo anterior, y teniendo en cuenta la necesidad de prevenir dichos eventos en Colombia, se reglamenta desde 1979 la implementación del programa de salud ocupacional, así mismo, se reitera dicha obligación y se regula la organización, funcionamiento y forma de los mismos, mediante la Resolución 1016 de 1989; posteriormente se adopta en el 2004 mediante la decisión 584 el instrumento Andino de seguridad y salud en el trabajo, este establece disposiciones que impulsan a los países miembros, como es el caso de Colombia, a la adopción de normas sobre sistemas de gestión de la seguridad y la salud en el trabajo (Decisión 584, 2004); por consiguiente, el Ministerio del Trabajo mediante la Ley 1562 de 2012, definió que el programa de salud ocupacional se entendería como el sistema de gestión de la seguridad y salud en el trabajo (Ley 1562, 2012) y cuyas disposiciones se definieron con la expedición del Decreto 1443 de 2014, acogido en el Decreto Único Reglamentario 1072 de 2015, el cual tiene como objeto anticipar, reconocer, evaluar y controlar los riesgos que puedan afectar a los trabajadores, mediante un proceso lógico y que incluya el mejoramiento continuo (Ministerio de Trabajo, 2017).

Por otra parte, la Ley 872 de 2003 establece el sistema de gestión de calidad obligatorio para organismos y entidades del sector central y descentralizado de la rama ejecutiva del poder público (Ley 872, 2003), adoptado para la gestión pública con la norma NTCGP 1000:2009 mediante el Decreto 4485 del 2009; estas disposiciones son derogadas por el Decreto 1499 de 2017 y en consecuencia se establece que los organismos tienen la potestad de decidir si se van a certificar bajo normas nacionales e internacionales de calidad 0 no, adicionalmente, señala que los certificados de la NTC GP1000:2009 continuarán vigentes hasta la fecha por la cual fueron expedidos (Decreto 1499, 2017).

De la misma forma, se debe tener en cuenta el impacto de la globalización, dado que esto ha impulsado a las organizaciones a la implementación de sistemas de gestión, que logren mejorar su competitividad e imagen, brinden mayor satisfacción a sus partes interesadas quienes cada vez son más exigentes, eviten fijar 
objetivos institucionales aislados, implementen cambios que generen rechazo debido a la dualidad de políticas, gastos innecesarios, duplicidad de funciones, repetición de procesos y procedimientos (Ahumada, 2014).

Por lo anterior, la metodología propuesta permitirá contribuir a las entidades públicas, a desarrollar sus actividades con calidad, y así mismo tomar medidas preventivas o correctivas frente a la protección y promoción de la salud de los trabajadores, teniendo en cuenta los accidentes de trabajo y la alta tasa de enfermedad laboral que presenta el sector público entre los años 2014 y 2015, como se muestra a continuación:

Tabla 1. Accidentalidad, enfermedad laboral y mortalidad en Colombia

\begin{tabular}{|c|c|c|c|c|c|}
\hline \multicolumn{2}{|c|}{$\begin{array}{c}2014 \\
\text { (Calificadas) }\end{array}$} & \multicolumn{3}{c|}{$\begin{array}{c}2015 \\
\text { (Calificadas) }\end{array}$} \\
\hline N. accidentes & N. enfermedades & $\begin{array}{c}\text { N. } \\
\text { muertes }\end{array}$ & N. accidentes & N. enfermedades & $\begin{array}{c}\text { N. } \\
\text { muertes }\end{array}$ \\
\hline 14.802 & 643 & 15 & 14.901 & 864 & 22 \\
\hline
\end{tabular}

Fuente: informe de indicadores del SGRL 2011-2015 (Direccion de Riesgos Laborales, 2016).

Por otro lado, esta metodología permitirá disminuir los problemas de los sistemas de gestión que se constituyen de forma independiente, quienes generan sobrecostos, teniendo en cuenta que se asignan recursos por separados; según Hurtado (2016), hoy en día las organizaciones ven la necesidad de integrar sistemas de gestión, principalmente de calidad, medio ambiental y de seguridad y salud en el trabajo, con el objetivo de optimizar recursos, reducir procesos y procedimientos, aumentar su eficacia, cumplir con la exigencias legales, superar las expectativas de sus partes interesadas, permitiendo mejorar su eficacia e incrementar su rentabilidad. Teniendo en cuenta lo definido, en un sistema de gestión que es como una persona donde cada parte del cuerpo, cumple con una actividad específica para un adecuado funcionamiento (Viloria, 2011).

Por consiguiente, es necesario conocer los costos que genera un sistema de gestión de calidad y que se pueden clasificar de la siguiente manera:

Tabla 2. Clasificación de costos de un sistema de gestión de calidad

\begin{tabular}{|l|l|l|}
\hline \multicolumn{1}{|c|}{ CLASIFICACIÓN } & \multicolumn{1}{|c|}{ TIPOS } & \multicolumn{1}{c|}{ DESCRIPCIÓN } \\
\hline \multirow{2}{*}{ Costos directos de conformidad } & Costos de prevención & $\begin{array}{l}\text { Inversión para prevenir fallas y lograr trabajos planificados con un alto } \\
\text { nivel de claridad. }\end{array}$ \\
\cline { 2 - 3 } & Costos de evaluación & $\begin{array}{l}\text { Claves para verificar que se están cumpliendo los requisitos y } \\
\text { requerimientos. }\end{array}$ \\
\hline \multirow{2}{*}{$\begin{array}{l}\text { Costos directos por no } \\
\text { conformidad }\end{array}$} & Costos de fallas externos & Se generan por causa de falencias en el servicio suministrado al usuario. \\
\cline { 2 - 3 } & Costos de fallos internos & $\begin{array}{l}\text { Generados por errores o imperfectos en los servicios antes de que estos } \\
\text { salgan. }\end{array}$ \\
\hline
\end{tabular}

Fuente: elaboración propia basado Perdomo (2001). 
Es por ello que se debe contemplar el balance entre los costos de protección a los trabajadores y los generados por falencias de la entidad, con el propósito de lograr incrementar la productividad y rentabilidad en las entidades (Perdomo, 2001).

De la misma manera, la implementación del SG-SST, conlleva a obtener beneficios en cuanto a costos relacionados con la atención e implementación de acciones para la prevención de accidentes e incidentes de trabajo, cumplimiento de la normativa legal vigente, un enfoque en el tiempo frente a la salud y seguridad de los trabajadores, interés de los usuarios para generar nuevos negocios y reducir los costos por incapacidades (Gómez, 2015).

Así mismo, se deben considerar los beneficios de integración (Iñaki, Merce y Martí, 2007) que sustentan las ventajas y desventajas relacionadas a la integración de sistemas de gestión, destacando las siguientes:

Tabla 3. Ventajas y desventajas de los sistemas integrados de gestión

\begin{tabular}{|l|l|}
\hline \multicolumn{1}{|c|}{ VENTAJAS } & \multicolumn{1}{|c|}{ DESVENTAJAS } \\
\hline - Simplificación de las exigencias del sistema. & - Dificultades para encontrar denominadores \\
- Optimización de los recursos. & comunes. \\
- Reducción de costes. & $\begin{array}{l}\text { Lada sistema de gestión. } \\
\text { - Realización de auditorías combinadas. }\end{array}$ \\
- Reducción de la documentación. & Miedo a la pérdida de trabajos por no tener \\
- Alineación de los objetivos de los distintos estándares y sistemas. & \\
- Creación de sinergias. & \\
- Reducción de duplicidad de documentetivos. \\
- Aumento de la motivación de los trabajadores. & \\
- Reducción de los conflictos. & \\
- Mejora de la efectividad y eficiencia de la entidad. & \\
\hline
\end{tabular}

Fuente: elaboración propia basada en el artículo de la integración de sistemas de gestión basados en estándares internacionales: (Iñaki, Merce y Martí, 2007).

Ahora bien, la norma PAS 99 elaborada por la British Standard Institution, se convierte en un marco para la integración de los requisitos comunes de los sistemas de gestión, y es una herramienta publica enfocada a las entidades que tienen el deseo o están implementando diferentes tipos de sistemas de gestión. A continuación un comparativo de esta norma con la UNE 66177:2005:

Tabla 4. Comparativo UNE 66177:2005 vs. PASS 99:2012

\begin{tabular}{|l|l|}
\hline \multicolumn{2}{|c|}{ GENERALIDADES } \\
\hline UNE 66177:2005 & \multicolumn{1}{c|}{ PAS 99:2012 } \\
\hline Integra los sistemas de gestión existentes en la organización. & Es una norma que especifica requisitos. \\
\hline Enfoque basado en procesos. & Basada en el anexo SL - Estructura de alto nivel. \\
\hline Es aplicable a cualquier tipo de organización. & Aplicable a todos los tipos de organización. \\
\hline No certificable. & Certificable. \\
\hline
\end{tabular}


Daissy Carolina Peralta Cruz y Schneider Guataquí Cervera

\begin{tabular}{|l|l|}
\hline \multicolumn{1}{|c|}{ UNE 66177:2005 } & \multicolumn{1}{c|}{ PAS 99:2012 } \\
\hline Involucra la evolución y el análisis del contexto de la organización. & Enfoque basado en procesos. \\
\hline $\begin{array}{l}\text { Es más adecuada en los casos donde se han implementado varios } \\
\text { sistemas de gestión, por separado y se tiene como propósito la } \\
\text { integración. }\end{array}$ & $\begin{array}{l}\text { Es más adecuada para las organizaciones que inician con un sistema de } \\
\text { gestión y tienen el propósito de implementar otros, de forma integrada. }\end{array}$ \\
\hline
\end{tabular}

Fuente: elaboración propia.

De acuerdo a lo anterior, el desarrollo de este proyecto se enfocó en los requisitos establecidos en la norma PAS 99, la cual en su versión 2012 tiene en cuenta la propuesta de la ISO, en cuanto a la articulación del ciclo PHVA y la adaptación a la estructura de alto nivel.

Por consiguiente, la metodología propuesta identifica los elementos comunes y articula los sistemas de gestión que la integran, basándose en la estructura de alto nivel con un enfoque basado en procesos, esta metodología contiene los elementos que permiten la implementación del sistema de gestión de la seguridad y salud en el trabajo en el sistema de gestión de calidad en las entidades del sector público colombiano. La cual puede ser implementada por todos los organismos y entidades del sector central y del sector descentralizado por servicios de la rama ejecutiva del poder público del orden nacional, y para el desarrollo de las funciones propias de las demás ramas del poder público en el orden nacional, permitiendo desarrollar sus actividades con calidad y así mismo tomar las medidas preventivas o correctivas frente a la protección y promoción de la salud de los trabajadores.

\section{METODOLOGÍA}

El proyecto de investigación se basa en métodos mixtos (cuantitativo y cualitativo) donde Castañeda, Prado y Vargas (2016), señalan que interactúan conjuntamente, con el propósito de tener una mejor comprensión de la investigación que se realiza. Esta combinación desarrolla el problema de interés desde diferentes opiniones y puntos de vista, permitiendo contrastar los resultados de ambos y lograr articularlos (Sampieri, Collado y Lucio, 2010).

Es por ello que este proyecto contempló los siguientes elementos cualitativos: revisión de la literatura sobre estrategias y herramientas para la integración de sistemas de gestión, análisis de correspondencia de los requisitos normativos y construcción de la propuesta metodológica para la integración del SG-SST en el SGC en las entidades públicas colombianas de orden nacional. En cuanto a los elementos cuantitativos se encuentra la validación contenido, esta fue desarrollada mediante la aplicación del instrumento, la tabulación, el procesamiento, análisis e interpretación de los datos provenientes de las encuestas a expertos.

Es decir, el pragmatismo en la investigación se llevó a cabo al iniciar con un proceso conceptual cualitativo, realizando la búsqueda de literatura que permitiera contribuir a la construcción de la metodología, para ello, se diseñó una matriz de consolidación de literatura, la cual tenía como variables las fechas de publicación, tipos de sistema de gestión en los que se centra la investigación (calidad, seguridad y salud en el trabajo o un sistema integrado de gestión), tipo de organización (pública o privada), alcance de los proyectos y referentes normativos. En esta búsqueda se tienen en cuenta tres aspectos fundamentales que fueron: los proyectos de investigación en la materia, estrategias y herramientas para la integración de sistemas de gestión. 
Una vez consolidado el documento, se identificó la literatura de mayor relevancia para el proyecto y se logró establecer los referentes teóricos, de integración y de validación, de la siguiente manera:

Tabla 5. Referentes de investigación

\begin{tabular}{|l|l|l|l|}
\hline \multicolumn{1}{|c|}{ TIPO } & \multicolumn{1}{|c|}{ REFERENTE } & \multicolumn{1}{c|}{ CRITERIO } & \multicolumn{1}{c|}{ DESCRIPCIÓN } \\
\hline \multirow{5}{*}{ Teórico } & OIT & $\begin{array}{l}\text { Seguridad y salud en el } \\
\text { trabajo }\end{array}$ & Directivas relativas a los SG-SST (ILO-OSH-2001) \\
\cline { 2 - 4 } & Merce y Martí & Beneficios de integración & Ventajas y desventajas \\
\cline { 2 - 5 } & $\begin{array}{l}\text { Plan nacional de } \\
\text { desarrollo, Ley 1752 de } \\
2015\end{array}$ & Innovación & $\begin{array}{l}\text { Sistema Único de Información de Riesgos Laborales. } \\
\text { Gestión frente a sus servicios y al fortalecimiento del } \\
\text { talento humano en cuanto la SST. } \\
\text { Competitividad e infraestructura estratégica, desarrollo } \\
\text { científico, tecnológico y la innovación. }\end{array}$ \\
\hline \multirow{2}{*}{ Integración } & PASS 99 & Estructura de alto nivel & $\begin{array}{l}\text { Ciclo PHVA } \\
\text { Estructura de alto nivel }\end{array}$ \\
\hline \multirow{2}{*}{ Validación } & Rodríguez-Rojas (2017) & Validación de contenido & Criterios: claridad, pertinencia y aplicabilidad \\
\cline { 2 - 5 } & Escobar y Cuervo & Pasos para la validación \\
\hline
\end{tabular}

Fuente: elaboración propia.

Paralelamente se desarrolló el análisis de correspondencia y sus resultados fueron consolidados en una matriz donde se visualizó de forma gráfica la integración de los requisitos establecidos en el Decreto 1072 de 2015, la Norma técnica de calidad para la gestión pública NTCGP 1000:2009 y la Norma técnica de calidad ISO 9001:2015. De igual manera, en esta matriz se relacionaron las diferentes metodologías y herramientas de gestión para la implementación de los requisitos integrables.

Tabla 6. Cuadro de correlación de requisitos

\begin{tabular}{|c|c|c|c|c|c|}
\hline \multicolumn{2}{|c|}{ DECRETO 10722015} & \multicolumn{2}{c|}{ GP 1000:2009 } & \multicolumn{2}{c|}{ ISO 9001:2015 } \\
\hline Numeral & Requisito & Numeral & Requisito & Numeral & Requisito \\
\hline & & & & & \\
\hline
\end{tabular}

Fuente: elaboración propia.

Con base a los resultados de la consulta de literatura y la elaboración de la correlación de requisitos, se documentó la metodología de integración del sistema de gestión de la seguridad y salud en el trabajo en el sistema de gestión de calidad, para las entidades públicas colombianas de orden nacional. Esta se estructuró basándose en las directrices relativas a los sistemas de gestión de seguridad y salud en el trabajo ILO-OSH 
2001, donde se identifican como elementos principales de la mejora continua la política, la organización, la planificación y aplicación, la evaluación y las acciones en pro de mejoras (Organizacion Internacional del Trabajo, 2001).

Una vez finalizada la fase cualitativa, se procedió a la etapa cuantitativa, con el propósito de realizar la validación, análisis e interpretación de los datos de acuerdo a los diferentes puntos de vista y opiniones de los expertos. Este proceso fue desarrollado en dos momentos, una validación con profesionales en microbiología industrial, administración de empresas, química farmacéutica, ingenierías, administración pública 0 afines y en formación como magíster en calidad y gestión integral, adicional, acreditaron 3 años de experiencia en procesos de gestión de calidad o seguridad y salud en el trabajo, el instrumento de validación fue remitido a 12 personas, de las cuales se recibió respuesta de 9 , adicional se realizó la validación con profesionales universitarios de (ingenierías, administración de empresas, administración pública o afines) 0 profesional especializado en sistemas de gestión o áreas afines; vinculados como servidores públicos en entidades públicas colombianas de orden nacional (específicamente ministerios), donde acreditaron 3 años de experiencia en procesos de gestión de calidad o seguridad y salud en el trabajo; obteniendo respuesta de 15 funcionarios pertenecientes a los siguientes ministerios (MinAmbiente, MinMinas, MinTIC, MinTrabajo y MinVivienda).

El instrumento utilizado para la validación fue basado en Rojas (2017), este contiene las siguientes características: objetivo, criterios divididos en tres variables (claridad, pertinencia y aplicabilidad), observaciones, sugerencias, nombre, formación, experiencia del experto, entidad y fecha de diligenciamiento.

Finalmente, una vez aplicado el instrumento de validación de contenido, se realizó la respectiva consolidación y análisis de los resultados.

\section{RESULTADOS Y DISCUSIÓN}

Este proyecto de investigación propone una metodología que puede ser implementada por todos los organismos y entidades de la rama ejecutiva del poder público del orden nacional y en la gestión administrativa necesaria para el desarrollo de las funciones propias de las demás ramas del poder público en el orden nacional.

El desarrollo de la metodología se basa en la nueva estructura de alto nivel propuesta por la ISO para los sistemas de gestión y contempla la integración de los sistemas de gestión de seguridad y salud en el trabajo y de calidad, con el fin de generar el conocimiento requerido y para facilitar la implementación de estos sistemas en entidades públicas de manera integrada. Esta metodología permite garantizar el cumplimiento normativo relacionado a los requisitos integrables del sistema de gestión de calidad para la gestión pública (NTC GP 1000:2009), el sistema de gestión de calidad (ISO 9001:2015) y el sistema de gestión de seguridad y salud en el trabajo de acuerdo establecido en el Decreto único Reglamentario del sector trabajo (libro 2, parte 2, título 4, capitulo 6).

La estructura de la metodología se definió teniendo en cuenta los requisitos que demandan integración y garantizando que se mantenga el enfoque del ciclo de mejora continua; para ello se definió una categoría central "sistema integrado de gestión" y como categorías los capítulos principales de las directivas relativas a los sistemas de gestión de seguridad y salud en el trabajo (ILO-OSH-2001), así mismo, se definieron subcategorías que incluyeron los requisitos identificados como integrables de acuerdo a lo que se muestra en la siguiente tabla: 
Tabla 7. Estructura de la propuesta metodológica

\begin{tabular}{|l|l|}
\hline \multicolumn{1}{|c|}{ Categoría } & \multicolumn{1}{c|}{ Subcategoría } \\
\hline Política integrada & Política integrada calidad y SST. \\
\hline \multirow{5}{*}{ Organización } & Rendición de cuentas. \\
\cline { 2 - 2 } & Obligación, responsabilidad, autoridad y comunicación. \\
\cline { 2 - 2 } & Capacitación, formación, competencia y toma de conciencia. \\
\cline { 2 - 2 } & Documentación. \\
\cline { 2 - 2 } & Comunicación interna y externa. \\
\hline \multirow{5}{*}{ Planificación y aplicación } & Evaluación inicial y planificación. \\
\cline { 2 - 3 } & Objetivos. \\
\cline { 2 - 3 } & Identificación de peligros, evaluación y valoración de los riesgos. \\
\cline { 2 - 3 } Evaluación & Medidas de prevención y control. \\
\cline { 2 - 3 } & Gestión del cambio. \\
\cline { 2 - 3 } & Adquisiciones y contratación. \\
\hline Acción en pro de mejoras & Seguimiento, evaluación, medición y análisis de mejora. \\
\hline & Auditoria interna. \\
\hline & Revisión por la alta dirección. \\
\hline & Acción correctiva, preventiva y de mejora. \\
\hline
\end{tabular}

Fuente: elaboración propia basada en la matriz de correlación de requisitos.

La descripción de cada subcategoría se basó en la correlación de requisitos, donde se muestra en primera instancia los requisitos integrables y relacionados entre el Decreto 1072 de 2015, la NTC GP 1000:2009 y la
NTC ISO 9001:2015, posteriormente se encuentran los ítems integrados y se listan las actividades identificadas como principales para la implementación de controles, como se muestra en la siguiente tabla:

Tabla 8. Controles integrados por categoría

\begin{tabular}{|l|l|}
\hline \multicolumn{1}{|c|}{ Subcategoría } & \multicolumn{1}{c|}{ Ítems } \\
\hline $\begin{array}{l}\text { Política integrada calidad y } \\
\text { SST }\end{array}$ & $\begin{array}{l}\text { Documentada, firmada, divulgada, disponible y aplicada. Con alcance a todos los trabajadores. Coherentes con } \\
\text { planes estratégicos. Revisión anual y mejora continua. Específica y apropiada a la empresa, tamaño, propósito y } \\
\text { contexto. Cumplimiento de requisitos CLIO. Establecer el compromiso. }\end{array}$ \\
\hline Rendición de cuentas & $\begin{array}{l}\text { Debe ser realizado por todos los que se les haya asignado responsabilidades en el SG-SST. Rendir cuentas con } \\
\text { relación al desempeño en SST. Se debe realizar mínimo una vez al año. Se puede realizar a través de medios } \\
\text { escritos, electrónicos, verbales o una integración de estos. Debe estar documentada. }\end{array}$ \\
\hline $\begin{array}{l}\text { Obligación, responsabilidad, } \\
\text { autoridad y comunicación }\end{array}$ & $\begin{array}{l}\text { La alta dirección debe definirlas, asignarlas comunicarlas y entenderlas, para empleadores y trabajadores. } \\
\text { Identificar las obligaciones de las ARL. }\end{array}$ \\
\hline
\end{tabular}




\begin{tabular}{|c|c|}
\hline Subcategoría & Ítems \\
\hline $\begin{array}{l}\text { Capacitación, formación, } \\
\text { competencia y toma de } \\
\text { conciencia }\end{array}$ & $\begin{array}{l}\text { Determinar la competencia necesaria de las personas. Definir requisitos de formación, conocimiento y práctica. } \\
\text { Toma de conciencia de las personas. Adoptar y mantener disposiciones para su cumplimiento, elaborar un } \\
\text { programa de capacitación, con revisión anual, todos los niveles de la entidad incluyendo a todos los trabajadores } \\
\text { independiente de su forma de contratación o vinculación, evaluar la eficacia de las acciones, conservar } \\
\text { documentación que dé cuenta de la competencia, participación del COPASST o vigía de SST, todo funcionario } \\
\text { que entre por primera vez, independiente de su forma de contratación y vinculación, de manera previa al inicio } \\
\text { de sus labores proporcionar una inducción. }\end{array}$ \\
\hline Documentación & $\begin{array}{l}\text { Mantener disponible y actualizados los documentos requeridos por las normas del sistema. Control de } \\
\text { documentos, en custodia del responsable del sistema de gestión, redactada de manera clara y entendible, } \\
\text { control de registros, conservar los registros requeridos por las normas del sistema, conservación de documentos } \\
\text { y registros. }\end{array}$ \\
\hline $\begin{array}{l}\text { Comunicación interna y } \\
\text { externa }\end{array}$ & $\begin{array}{l}\text { Recibir, documentar y responder comunicaciones internas o externas. Tener mecanismos de comunicación } \\
\text { externa e interna. }\end{array}$ \\
\hline $\begin{array}{l}\text { Evaluación inicial y } \\
\text { planificación }\end{array}$ & $\begin{array}{l}\text { Contexto de la organización. Partes interesadas. Identificar prioridades en el SST. Autoevaluación del sistema } \\
\text { de conformidad con la normatividad vigente, documentada. Mecanismos de planificación basados en la } \\
\text { evaluación inicial. Recursos, riesgos y oportunidades del sistema. Acciones para abordar riesgos y oportunidades. }\end{array}$ \\
\hline Objetivos & $\begin{array}{l}\text { Ser claros, medibles, cuantificables y tener metas. Adecuados para las características, el tamaño y la actividad } \\
\text { económica de la empresa. Compatibles con la normatividad aplicable. Comunicados, revisados y evaluados } \\
\text { mínimo una vez al año. Pertinentes para la conformidad de los productos y servicios, documentados. }\end{array}$ \\
\hline $\begin{array}{l}\text { Identificación de peligros, } \\
\text { evaluación y valoración de los } \\
\text { riesgos-medias de prevención } \\
\text { y control }\end{array}$ & $\begin{array}{l}\text { Aplicar una metodología sistemática, documentada. Utilizar metodologías adicionales para complementar la } \\
\text { evaluación de los riesgos. Informar al COPASST o vigía de SST resultados de las evaluaciones ambientales. } \\
\text { Identificar y relacionar los trabajadores de actividades de alto riesgo. Empleador debe suministrar los EPP. } \\
\text { Realizar mantenimiento de las instalaciones, equipos y herramientas. Realizar acciones de vigilancia de la salud } \\
\text { de los trabajadores. Corregir condiciones inseguras. }\end{array}$ \\
\hline Gestión del cambio & $\begin{array}{l}\text { Procedimiento para evaluar el impacto sobre SST que puedan generar los cambios internos o externos. } \\
\text { Peligros y evaluación de riesgos derivados de estos cambios. Informar y capacitar a los trabajadores sobre los } \\
\text { cambios. Determinar necesidad del cambio. Propósito y consecuencias. Integridad del Sistema. Recursos. } \\
\text { Responsabilidades y autoridades. Identificar cambios en requisitos de productos o servicios. Revisar y controlar } \\
\text { cambios en la producción y prestación del servicio. }\end{array}$ \\
\hline Adquisiciones y contratación & $\begin{array}{l}\text { Procedimiento para evaluar el impacto sobre SST que puedan generar los cambios internos o externos. } \\
\text { Peligros y evaluación de riesgos derivados de estos cambios. Informar y capacitar a los trabajadores sobre los } \\
\text { cambios. Determinar necesidad del cambio. Propósito y consecuencias. Integridad del Sistema. Recursos. } \\
\text { Responsabilidades y autoridades. }\end{array}$ \\
\hline $\begin{array}{l}\text { Seguimiento, evaluación, } \\
\text { medición y análisis de mejora }\end{array}$ & $\begin{array}{l}\text { Identificar que necesita seguimiento y medición. Métodos y periodicidad apropiados para el seguimiento de } \\
\text { los procesos, efectividad y manejo de recursos. Correcciones, acciones correctivas o preventivas. Resultados } \\
\text { disponibles y difundidos de manera permanente. Evaluar desempeño y eficacia del Sistema. Conservar } \\
\text { información documentada. }\end{array}$ \\
\hline Auditoria interna & $\begin{array}{l}\text { Procedimiento y registros. Debe realizarse en intervalos planificados, pero como mínimo una vez al año. } \\
\text { Planificada con la participación del COPASST. Elaboración del programa de auditoría. Definición de criterios } \\
\text { y alcance de la auditoria. Selección de auditores. Realizar auditorías asegurando objetividad e imparcialidad. } \\
\text { Comunicar los resultados de auditoría. Responsable del proceso debe asegurar la toma de acciones frente a los } \\
\text { resultados de la auditoría. }\end{array}$ \\
\hline Revisión por la alta dirección & $\begin{array}{l}\text { Procedimiento y registros. Debe realizarse en intervalos planificados, pero como mínimo una vez al año. } \\
\text { Planificada con la participación del COPASST. Elaboración del programa de auditoría. Definición de criterios } \\
\text { y alcance de la auditoria. Selección de auditores. Realizar auditorías asegurando objetividad e imparcialidad. } \\
\text { Comunicar los resultados de auditoría. }\end{array}$ \\
\hline
\end{tabular}




\begin{tabular}{|c|c|}
\hline Subcategoría & Ítems \\
\hline $\begin{array}{l}\text { Acción correctiva, preventiva } \\
\text { y de mejora }\end{array}$ & $\begin{array}{l}\text { A partir de resultados de supervisión y medición, auditorías, revisión por la dirección y quejas. Documentadas } \\
\text { y difundidas. Definición de responsables y fechas de cumplimiento. Identificar oportunidades de mejora } \\
\text { continua. Garantizar los recursos para la mejora continua. Procedimiento acciones correctivas, preventivas. } \\
\text { Conservar información documentada. }\end{array}$ \\
\hline
\end{tabular}

Fuente: elaboración propia.

Finalmente se proponen y se describen las actividades y herramientas necesarias para la implementación de los requisitos integrables de los sistemas de gestión en mención.

Es importante precisar que dentro de la categoría de planificación y aplicación, se incluyó como subcategoría la evaluación inicial, debido a su importancia para identificar las prioridades de seguridad y salud en el trabajo, y teniendo en cuenta su correlación con el requisito de contexto estratégico del sistema de gestión de calidad, no obstante se tuvo en cuenta lo establecido en los artículos 2.2.4.6.16 y 2.2.4.6.37 del Decreto 1072 de 2015, donde se determina que la autoevaluación debe incluir los estándares mínimos que fueron reglamentados mediante la Resolución 1111 de 2017, es por ello que el anexo técnico señalado en dicha resolución fue incluido dentro de la caja de herramientas de la guía, y se convierte en un insumo fundamental para verificar, planificar y dar cumplimiento de los requisitos del sistema de gestión de la seguridad y salud en el trabajo.

De acuerdo con lo anterior, esta metodología genera innovación en la integración de sistemas de gestión, ya que se basa en la nueva estructura de alto nivel propuesta por la ISO para los sistemas de gestión, y mantiene los criterios del ciclo de mejora (planear, hacer, verificar y actuar) el cual está contemplado desde las directrices de la OIT.

Por otra parte, se identificaron dentro de la metodología requisitos no integrables, debido a su especificidad y particularidad, tal como se muestra en las siguientes tablas:

Tabla 9. Requisitos no integrables del SGC NTC GP 1000:2009 e ISO 9001:2015

\begin{tabular}{|c|l|c|l|}
\hline \multicolumn{2}{|c|}{ GP 1000:2009 } & \multicolumn{1}{c|}{ ISO 9001:2015 } \\
\hline N. ${ }^{\circ}$ & \multicolumn{1}{|c|}{ Requisito } & \multicolumn{2}{c|}{ Requisito } \\
\hline 4 & Sistema de gestión de la calidad & 4,4 & Sistemas de gestión de la calidad y sus procesos \\
\hline 4.1 & Requisitos generales & & \\
\hline 4.2 .2 & Manual de calidad & 4.3 & Determinar el alcance del sistema de gestión de la calidad \\
\cline { 1 - 2 } 5.2 & Enfoque al cliente & 5.1 .2 & Enfoque al cliente \\
\hline 7 & Realización del producto o prestación del servicio & 8 & Operación \\
\hline 7.1 & Planificación de la realización del producto o prestación & 8.1 & Planificación y control operacional \\
\hline
\end{tabular}


Daissy Carolina Peralta Cruz y Schneider Guataquí Cervera

\begin{tabular}{|c|c|c|c|}
\hline \multicolumn{4}{|c|}{ SGC } \\
\hline \multicolumn{2}{|r|}{ GP 1000:2009 } & \multicolumn{2}{|r|}{ ISO 9001:2015 } \\
\hline 7.2 & Procesos relacionados con el cliente & 8.2 & Requisitos para productos y servicios \\
\hline 7.2 .1 & $\begin{array}{l}\text { Determinación de los requisitos relacionados con el } \\
\text { producto y/o servicio }\end{array}$ & 8.2 .2 & $\begin{array}{l}\text { Determinación de los requisitos para los productos y } \\
\text { servicios }\end{array}$ \\
\hline 7.2 .2 & $\begin{array}{l}\text { Revisión de los requisitos relacionados con el producto } \\
\text { y/o servicio }\end{array}$ & 8.2 .3 & Revisión de los requisitos para los productos y servicios \\
\hline 7.3 & Diseño y desarrollo & 8,3 & Diseño y desarrollo de productos y servicios \\
\hline 7.3.1 & Planificación del diseño y desarrollo & $\begin{array}{l}8.3 .1 \\
8.3 .2\end{array}$ & $\begin{array}{l}\text { Generalidades } \\
\text { Planificación del diseño y desarrollo }\end{array}$ \\
\hline 7.3 .2 & Elementos de entrada para el diseño y desarrollo & 8.3 .3 & Entradas para el diseño y desarrollo \\
\hline 7.3.3 & Resultados del diseño y desarrollo & 8.3 .5 & Salidas para el diseño y desarrollo \\
\hline 7.3 .4 & Revisión del diseño y desarrollo & \multirow{3}{*}{8.3 .4} & \multirow{3}{*}{ Controles del diseño y desarrollo } \\
\hline 7.3 .5 & Verificación del diseño y desarrollo & & \\
\hline 7.3.6 & Validación del diseño y desarrollo & & \\
\hline 7.3 .7 & Control de los cambios del diseño y desarrollo & 8.3 .6 & Cambios del diseño y desarrollo \\
\hline 7.5 & Producción y prestación del servicio & 8.5 & Producción y prestación del servicio \\
\hline 7.5.1 & Control de la producción y de la prestación del servicio & $\begin{array}{l}8.5 .1 \\
8.5 .5\end{array}$ & $\begin{array}{l}\text { Control de la producción y de la prestación del servicio } \\
\text { actividades posteriores a la entrega }\end{array}$ \\
\hline 7.5 .2 & $\begin{array}{l}\text { Validación de los procesos de la producción y de la } \\
\text { prestación del servicio }\end{array}$ & 8.5 .1 & Control de la producción y de la prestación del servicio \\
\hline 7.5.3 & Identificación y trazabilidad & 8.5 .2 & Identificación y trazabilidad \\
\hline 7.5.4 & Propiedad del cliente & 8.5.3 & Propiedad de los clientes o proveedores externos \\
\hline 7.5.5 & Preservación del producto y/o servicio & 8.5 .4 & Preservación \\
\hline 8.2 .1 & Satisfacción del cliente & 9.1 .2 & Satisfacción del cliente \\
\hline 8.2 .4 & Seguimiento y medición del producto y/o servicio & 8.6 & Liberación de productos y servicios \\
\hline 8.3 & Control del producto y/o servicio no conforme & 8.7 & Control de las salidas no conformes \\
\hline
\end{tabular}

Fuente: elaboración propia.

Tabla 10. Requisitos no integrables del SG-SST Decreto 1072 de 2015

\begin{tabular}{|l|l|}
\hline \multicolumn{2}{|c|}{ SG-SST } \\
\hline \multicolumn{1}{|c|}{ Decreto 1072 de 2015 } \\
\hline Artículo 2.2.4.6.32 & Investigación de incidentes, accidentes de trabajo y enfermedades laborales \\
\hline Artículo 2.2.4.6.25 & Prevención, preparación y respuesta ante emergencias \\
\hline
\end{tabular}

Fuente: elaboración propia. 
Sin embargo, se incluyó el requisito no integrable referente a la gestión de los peligros y riesgos, debido a su importancia e impacto en las condiciones laborales, así como su contribución a la prevención de accidentes y enfermedades por ocasión al trabajo; además de los diferentes requisitos integrables, como por ejemplo, la política, la gestión del cambio, los indicadores, las acciones preventivas y correctivas, entre otros, que promueven la protección y promoción de la salud de los trabajadores, así mismo ayudan a forjar ambientes de trabajos seguros y saludables.

Además, con el propósito de realizar la validación de contenido de la propuesta metodológica, se tomó como referente a Pérez y Martínez (2008), quienes establecen una guía para realizar el juicio de expertos, mediante la definición del objetivo, selección de expertos, explicitar las dimensiones, diseño del instrumento, medir la concordancia de los expertos y elaborar las conclusiones.

Como resultado, la muestra para la validación de este proyecto se determinó de manera intencional en las entidades de la rama ejecutiva del poder público en el orden nacional, específicamente ministerios, con el fin de determinar el alcance con entidades de similares características en la prestación de su servicio, ya que son las entidades encargadas de la formulación de políticas públicas e instrumentos normativos para el territorio nacional de acuerdo a su misionalidad.

La validación de contenido fue realizada a 9 de 12 expertos en formación como magíster en calidad y gestión integral de la Universidad Santo Tomás de Colombia, y a 15 de 16 expertos de los ministerios. $\mathrm{Al}$ realizar la unificación de los resultados, es decir las 24 validaciones, se identificó que el $89 \%$ de las personas participantes están de acuerdo y totalmente de acuerdo, lo que evidenció en la metodología un alto porcentaje de claridad, pertenencia y aplicabilidad. Es importante resaltar que los resultados obtenidos en parcialmente de acuerdo son neutros, por lo tanto, no se contemplaron para la toma de decisiones.

Fiǵura 1. Resultados de la validación (metodología)

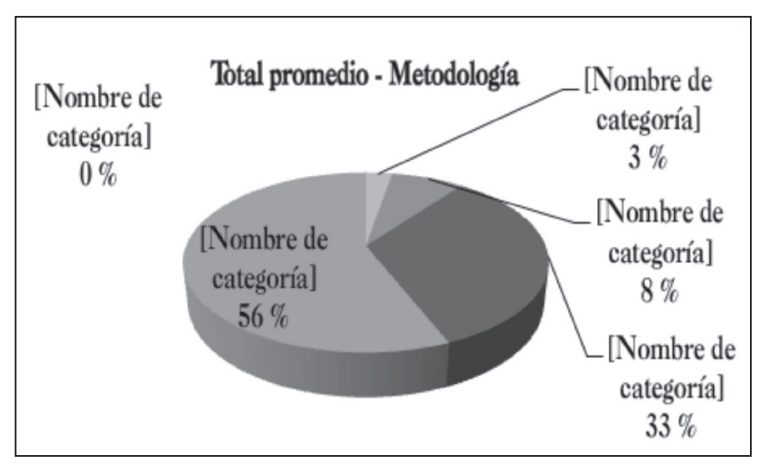

Fuente: elaboración propia.

El instrumento de validación aplicado estableció tres dimensiones: claridad, pertinencia y aplicabilidad; las cuales obtuvieron las siguientes deducciones:

Con relación a la claridad de la información, se obtienen resultados satisfactorios, ya que el $96 \%$ de los participantes se encuentran totalmente de acuerdo y de acuerdo, solo un $4 \%$ se encuentra parcialmente de acuerdo.

Fiǵura 2. Total promedio de la dimensión (claridad)

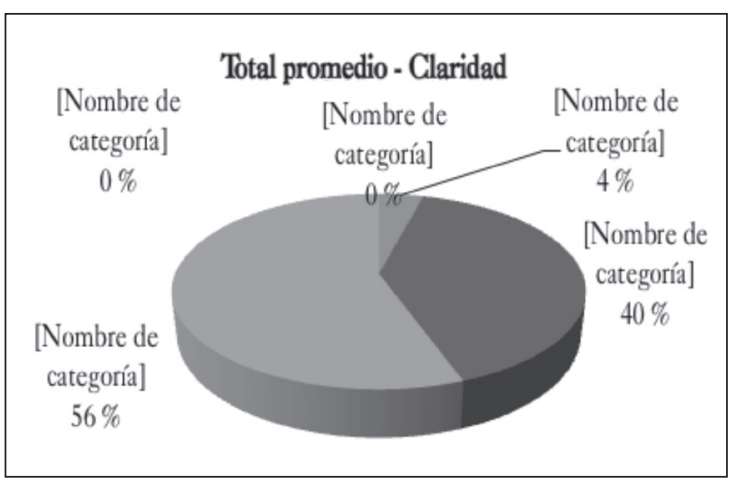

Fuente: elaboración propia.

Un $86 \%$ de los encuestados establece que la metodología es pertinente y el 14 \% están parcialmente de acuerdo y en desacuerdo. De igual manera, se 
identifica que el ítem que genera desacuerdo es la aplicabilidad de la metodología en el sector privado.

Figura 3. Total promedio de la dimensión (pertinencia)

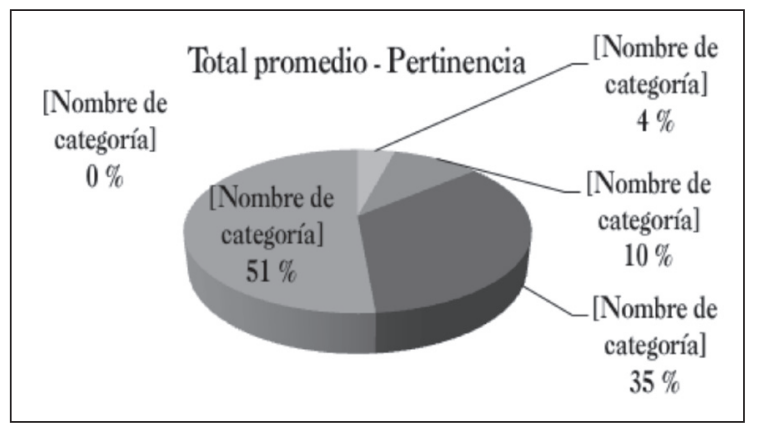

Fuente: elaboración propia.

Finalmente, con relación a la dimensión de aplicabilidad, se identifica un porcentaje alto de satisfacción, con un $90 \%$ de los encuestados de acuerdo y totalmente de acuerdo y un $10 \%$ parcialmente de acuerdo y en desacuerdo.

Figura 4. Total promedio de la dimensión (aplicabilidad)

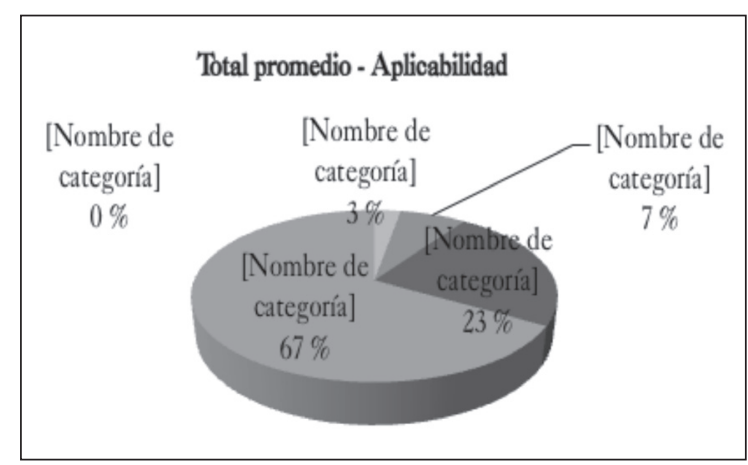

Fuente: elaboración propia.

Con el fin de determinar la necesidad de modificaciones de la metodología, se hizo necesario realizar una matriz de consolidación de resultados, en la cual se relacionó el nombre del experto, la entidad donde trabaja, las observaciones generales de la metodología y las sugerencias, estas últimas se clasifican como positivas, negativas y no aplica. Las clasificadas como positiva son aquellas que no relacionan cambios en la metodología, las negativas son las que proponen modificaciones y mejoras en la descripción de la metodología y las clasificadas como no aplica, son aquellas que, de acuerdo al análisis de los investigadores, se consideraron imparciales para la mejora de la metodología o no fueron claras.

Los resultados de la validación de la metodología por los expertos, nos permitieron considerar la aplicación de la metodología a cualquier tipo de entidad pública, y no solo a las de orden nacional de la rama ejecutiva como se estableció en el alcance del proyecto. Adicionalmente, es aplicable a organizaciones del sector privado, ya que contempla los requisitos del Decreto 1072 de 2015, el cual es de obligatorio cumplimiento y la norma ISO 9001:2015, la cual aplica a cualquier tipo de organización. Considerando de igual manera que los controles propuestos se identifican a través de metodologías y herramientas de gestión recopiladas en el análisis de literatura, basado para el sector público y privado.

Así mismo, esta metodología brinda beneficios en cuanto al cumplimiento de sus requisitos de forma integrada, como son: la alineación de los objetivos, el mejoramiento de la productividad y la prestación del servicio, la optimización de la operación de los procesos, la reducción de las interrupciones en el trabajo y reprocesos, el mejoramiento de la relación y la comunicación con las partes interesadas y la prevención de sobrecostos y sanciones administrativas, las cuales son directamente relacionadas a las ventajas sustentadas por Iñaki, Merce y Martí (2007).

Para dar continuidad a esta metodología y generar valor adicional a las entidades públicas, se recomienda contemplar la actualización del modelo integrado de planeación y gestión, adoptado mediante el Decreto 1499 de 2017, en donde se establece que las entidades y organismos públicos podrán considerar pertinente 0 no certificarse bajo las normas nacionales e internacionales de calidad, además tener en cuenta que las 
certificaciones otorgadas de la NTCGP 1000 versión 2009, continúan vigentes hasta la fecha en la cual fueron expedidas (Decreto 1499, 2017).

\section{CONCLUSIONES}

La metodología propuesta identifica los elementos para la integración y dirección de las entidades públicas de orden nacional, para dar cumplimiento a la normatividad aplicable relacionada con la implementación de sus sistemas de gestión, específicamente en seguridad y salud en el trabajo conforme a lo establecido en el Decreto 1072 de 2015 y calidad bajo la NTCGP 1000:2009, teniendo un enfoque basado en la estructura de alto nivel contemplada en la norma técnica ISO 9001:2015. Entre estos elementos integrables se contempla el liderazgo, roles, responsabilidades y autoridades, política, objetivos, recursos, conocimiento, formación y toma de conciencia, información documentada, comunicación, contexto, medición, auditoría interna, mejora y revisión por la dirección.

De igual manera, esta metodología facilita la gestión de las entidades en el marco del sistema integrado de gestión, ya que aporta conocimiento técnico desarrollado a través de las herramientas de gestión propuestas, que contribuyen al cumplimiento de los requisitos, aportando habilidad a los profesionales designados y a los demás responsables del mismo, como líderes de su implementación en las entidades.

Por otra parte, se alcanzaron los objetivos propuestos, debido a que se desarrollaron las etapas planteadas y se logró de manera oportuna generar los productos esperados, como se muestra a continuación:

- Se desarrolló la matriz de correlación de requisitos, la cual permite identificar el alcance de la metodología propuesta en cuanto a los requisitos integrables que se enmarcan en el ciclo de mejora continua a través del contexto de la organización, riesgos y oportunidades, liderazgo, comunicación, ambientes de trabajo, control de suministros, auditorías, revisión por la dirección, mejora continua, entre otros.

- Se incluyeron las herramientas que permiten la implementación de los requisitos integrables, estas fueron recopiladas con base a la revisión de literatura y teniendo en cuenta la experiencia relacionada de los investigadores.

- Se documentó la metodología, la cual contempla los elementos necesarios para la implementación de un sistema integrado de gestión en las entidades públicas colombianas de orden nacional bajo los requisitos de las normas ISO 9001:2001, NTCGP 1000:2009 y el Decreto 1072 de 2015.

- Se desarrolló el proceso de validación de contenido con expertos y de acuerdo al análisis efectuado, se realizaron las modificaciones pertinentes. Así mismo, se identificó de manera satisfactoria la validez de la de metodología en las entidades públicas de orden nacional, garantizando la integración de los sistemas de gestión.

En el desarrollo de la metodología se contemplaron los principales aspectos para el proceso de integración, según lo establecido por Merce (2014) de la siguiente manera:

En cuanto a la estrategia de integración se refiere a la secuencia, el orden o las alternativas de implementación de los sistemas de gestión, la cual para este proyecto se adoptó con base a los requisitos del sistema de gestión de la seguridad y salud en el trabajo del Decreto 1072 de 2015, el sistema de gestión de calidad ISO 9001:2015 y adicionalmente la NTC GP 1000:2009. 
Para la metodología de integración, se involucraron las herramientas o modelos que permiten lograr un sistema integrado de gestión, mediante la elaboración de la caja de herramientas, producto de la consulta de literatura y experticia de los investigadores.

El nivel de integración de la metodología propuesta es parcial, puesto que, en la matriz de correlación, se identificaron los requisitos comunes de las normas objeto de investigación y con base a ello se determinó el alcance de la misma.

Con relación a las dificultades para la implementación de un sistema integrado de gestión, se identificaron la falta de recursos, las propias de la organización y las diferencias de las normas. Esta metodología busca realizar las actividades con el mínimo de recursos necesarios para su gestión.

En cuanto a los beneficios de la integración, busca principalmente el aumento de la eficiencia en las entidades, reflejados en la optimización de las actividades, como los recursos, evitando reprocesos y manejando los enfoques de manera integrada, así mismo, permite cumplir los requisitos legales, evitando sanciones administrativas, teniendo en cuenta que el incumplimiento de normas de seguridad y salud en el trabajo y aquellas obligaciones del empleador, previstas en el sistema general de riesgos laborales acarrean multas de hasta 500 salarios mínimos mensuales legales vigentes, de acuerdo a la gravedad de su incumpliendo. Sin perder de vista que, el no reporte de los accidentes o enfermedades laborales, así como en caso de generarse un accidente que cause la muerte al trabajador, acarreará multas de hasta 1000 salarios mínimos mensuales legales vigentes.

Esto permitirá que los recursos destinados por la administración para la implementación de los sistemas de gestión serán más eficientes, debido a que se podrán realizar de forma conjunta actividades como auditorias, procesos de contratación o selección de personal, procesos de capacitación, entre otras, por otro lado, se previenen gastos médicos por incapacidades o interrupciones de trabajo; evitando así sobrecostos al interior de la entidad.

Además, de la integración de los conceptos desde el contexto estratégico y la gestión de riesgos y oportunidades dirigidos a la seguridad y salud en el trabajo y calidad, implementará ambientes de trabajos seguros y saludables, de forma que se reduzcan los accidentes de trabajo y enfermedades diagnosticadas, disminuyendo las incapacidades o rehabilitaciones, impactando directamente la productividad y la prestación del servicio.

Así mismo, la integración de estos requisitos ayudará a la simplificación de procesos documentales, evitando la duplicidad de documentos y registros que tienen una misma finalidad, como por ejemplo, informes de auditorías o revisiones por la alta dirección, formatos de gestión del cambio, rendiciones de cuentas, acciones correctivas o preventivas, entre otros.

Es importante resaltar que de igual manera la alta dirección podrá realizar un seguimiento conjunto a los sistemas de gestión, lo cual facilita el análisis de la información y la toma de decisiones en la entidad, de tal forma que desde la planificación se contemplen las actividades necesarias que satisfagan las necesidades de las partes interesadas, como estar involucradas desde la política del SIG, acceder a la información, comunicación eficaz, participar en la rendición de cuentas y en la toma de decisiones cuando sea pertinente.

Todo lo anterior permitirá mejorar la imagen institucional de la entidad y el prestigio de esta, frente a sus partes interesadas. Además, esta metodología fue visualizada para la rama ejecutiva, pero se convierte en una herramienta base para la implementación de los sistemas de gestión en las ramas legislativa y judicial.

Es relevante tener en cuenta que los compromisos del país desde el Plan Nacional de Desarrollo establecidos 
en la Ley 1753 de 2015, contemplan un enfoque de innovación a la gestión, frente a sus servicios y al fortalecimiento del talento humano en cuanto la seguridad y salud en el trabajo con los beneficios relacionados, lo que hace que esta metodología fortalezca la gestión institucional de las entidades públicas (Ley 1753, 2015).

Adicionalmente, en el ejercicio del estado a través del Departamento Administrativo de la Función Pública y en su función de garantizar el fortalecimiento institucional en las entidades públicas, a través del Decreto 1499 de 2017, mediante el cual actualiza el modelo integrado de planeación y gestión, el cual integra los sistemas de desarrollo administrativo creados mediante el artículo 133 de la Ley 1753 de 2015 y el sistema de gestión de la calidad, deroga la NTC GP1000. Lo anterior, y desde la entrada en vigencia de dicho Decreto, evidencia que la norma técnica sigue siendo un componente administrativo en la gestión de las entidades públicas. Igualmente, este Decreto en su artículo 2.2.22.3.12 establece que las certificaciones otorgadas por la NTC GP1000 continuarán vigentes hasta la fecha para la cual fueron expedidas (Decreto 1499, 2017), lo que genera la pertinencia y vigencia de esta metodología en relación con los requisitos que contempla la norma.

\section{REFERENCIAS}

Ahumada, D. P. (2014). Modelo para la implmentación de un sistema de gestión integral alineado a la estrategia empresarial de la organización. (Trabajo de ensayo). Universidad Militar Nueva Granada. Recuperado de http://repository.unimilitar.edu. co/bitstream/10654/13842/2/RojasAhumada2015_ SistemaGesionIntegrado.pdf

Castañeda, R. G., Prado, M. L., y Vargas, M. O. (2016). Reflexión crítica epistemológica sobre métodos mixtos. Enfermería Universitaria, 248.
Decisión 584. (7 de mayo de 2004). Sustitución de la Decisión 547. Instrumento andino de seguridad y salud en el trabajo. Guayaquil, Ecuador: Comunidad Andina.

Decreto 1499. (11 de septiembre de 2017). Por medio del cual se modifica el Decreto 1083 de 2015, Decreto Único Reglamentario del Sector Función Pública, en lo relacionado con el Sistema de Gestión establecido en el artículo 133 de la Ley 1753 de 2015. Diario Oficial n. : 50.353. Bogota, Colombia: Congreso de la República.. Recuperado de http://www.alcaldiabogota.gov.co/sisjur/normas/Norma1.jsp?i=71261

Dirección de Riesgos Laborales. (2016). Indicadores del sistema general de riesgos laborales, cifras 2011-2015. Bogota.

Gómez, I. C. (2006). Una revisión a la luz de las nuevas condiciones del trabajo. Unio. Psychol, 106.

Gómez, E. S. (2015). Relación costo-beneficio en la implmentación de un sistema de gestion en seguridad y sahud en el trabajo. (Trabajo de ensayo). Universidad Militar Nueva Granada. Recuperado de http://repository.unimilitar.edu.co/bitstream/10654/6570/1/ Ensayo\%20final\%20OHSAS\%202015.pdf

Hurtado, P. A. (2016). Los sistemas integrados de gestion, estrategia organizacional y su importancia. Tecnologia FITEC, 6, 2.

Iñaki, H. S., Merce, B., y Martí, C. (2007). La integración de sistemas de gestión basados en estándares internacionales. Dirección y Administración de Empresas, (14), 164.

Ley 872. (30 de diciembre de 2003). Por la cual se crea el sistema de gestión de la calidad en la Rama Ejecutiva. Diario Oficial n.: 45.418. Bogotá, Colombia: Congreso de la República. Recuperado de http:// www.alcaldiabogota.gov.co/sisjur/normas/Norma1.jsp?i=11232 
Ley 1562. (11 de julio de 2012). Por la cual se modifica el sistema de riesgos laborales y se dictan otras disposiciones en materia de salud ocupacional. Diario Oficial n. : 48.488 Bogota, Colombia: Congreso de la República. Recuperado de http://www.alcaldiabogota.gov.co/sisjur/normas/Norma1.jsp?i=48365

Ley 1753. (9 de junio de 2015). Por la cual se expide el Plan Nacional de Desarrollo 2014-2018. Todos por un nuevo país. Diario Oficial n.: 49.358. Bogota, Colombia: Congreso de la República. Recuperado de http://www.alcaldiabogota.gov.co/sisjur/normas/Norma1.jsp?i=61933

Merce, B. (2014). Integration of management systems as an innovation a proposal a new model. Elsevier Ltd., 262.

Ministerio de Trabajo. (17 de enero de 2017). MinTrabajo amplía plazo para implementar el sistema de gestión de la seguridad y salud en el trabajo. Recuperado de http://www.mintrabajo.gov.co/prensa/ comunicados/2017/enero/mintrabajo-amplia-plazo-para-implementar-el-sistema-de-gestion-dela-seguridad-y-salud-en-el-trabajo

Organizacion Internacional del Trabajo. (2001). Directrices relativas a los sistemas de gestión y la seguridad y salud en el trabajo - ILO-OSH 2001. Recuperado de http://www.ilo.org/wcmsp5/groups/ public/---ed_protect/---protrav/---safework/documents/normativeinstrument/wcms_112582.pdf

Organizacion Internacional del Trabajo. (2011). Acerca de la OIT. Orígenes e historia. Recuperado de http:// www.ilo.org/global/about-the-ilo/history/lang--es/ index.htm

Perdomo, A. (2001). Administración de los costos de la calidad: medición del rendimiento de la calidad sobre la inversión : ¿vale la pena un sistema de calidad? Bogotá: Icontec.

Pérez, J. E., y Martínez, A. C. (2008). Validez de contenido y juicio de expertos: una aproximación a su utilización. Avances en Medición, (6), 30.

Rojas, Y. R. (2017). Evaluación de la madurez de la gestión de la seguridad y salud en el trabajo en universidades con acreditación de alta calidad. (Doctorado en Administración). Bogota, México: Universidad de Celaya.

Sampieri, H., Collado, C. F., y Lucio, M. D. (2010). Metodología de la investigación. En J. M. Chacón (Ed.). México: McGraw-Hill.

Viloria, S. G. (2011). Sistemas integrados de gestión, un reto para las pequeñas y medianas empresas. Escenarios, 9(1), 76. 